\title{
Atypical Location of Coumel Tachycardia in Adult: Case Report
}

\author{
Raoni de Castro Galvão ${ }^{1, *}$, João Paulo Velasco Pucci1 ${ }^{1}$, Ofir Gomes Vieira1
}

ORCID IDs

Galvão RC (D) https://orcid.org/0000-0001-5541-2684

Pucci.JPV (D) https://orcid.org/0000-0001-8925-9622

Vieira OG (D) https://orcid.org/0000-0002-8037-8681

\begin{abstract}
Coumel Tachycardia or Incessant Junctional Reentrant (IJRT) generally affects the infant-juvenile population and is characterized by tachycardia due to atrioventricular reentry (AV) mediated by an exclusive, slow, decremental retrograde conduction pathway mostly located in the posterosseptal region of the tricuspid ring. Case report: The present study reported the case of an adult, 49 years old, with IJRT of atypical location. The patient reported feeling palpitations for 5 years with worsening in the last 6 months. After one of his seizures, an electrocardiogram (ECG) documented tachycardia due to AV reentry with a long RP 'interval. Subjected to an electrophysiological study, an accessory pathway of exclusive retrograde conduction with decreasing characteristics was observed, located in the right mid-septal region. There was easy induction of IJRT, remaining sustained throughout the study. A radiofrequency ablation was performed in the mid-septal D region with interruption of tachycardia, with no more atrioventricular retrograde conduction, the procedure ended without complications. Discussion: Interestingly, the case described differs both in terms of the age range of the most frequent involvement of the IJRT (children and young people), as well as the most common location of the accessory pathway (posterior and postero-septal region of the tricuspid ring). Ablation proved to be effective in this case and is the treatment of choice for IJRT despite the anomalous location of the accessory pathway (Middle-septal D: described in bibliographic reviews in only $7 \%$ of IJRT cases) and a potentially dangerous region due to proximity to the trunk of the His beam.
\end{abstract}

KEYWORDS: Catheter ablation; Reciprocating tachycardia; Supraventricular tachycardia

1. Centro de Ritmologia de Brasília - Brasília (DF), Brazil.

*Correspondonding author: raoni.castrogalvao@yahoo.com.br

Received: May 25, 2020 | Accepted: Jun 12, 2020 


\section{INTRODUCTION}

Incessant Junctional Reentrant Tachycardia (IJRT), also known as Coumel Tachycardia, is a supraventricular tachycardia by atrioventricular $(\mathrm{AV})$ reentry $(\mathrm{AV})$ mediated by an exclusive and slow retrograde conduction pathway, with diminishing characteristics, located mainly in the posterosseptal region of the tricuspid ring. Electrocardiographically, it manifests as a narrow $\mathrm{QRS}$ tachycardia with long RP', with negative retrograde "p" waves in lower leads (D2, D3 and avF). The child and youth population is generally the most affected by this disease; however, although unusual, it is possible to find adults and even the elderly with this tachycardia ${ }^{1-6}$.

Due to its incessant characteristic in some cases, patients may present tachycardiomyopathy, with myocardial dilation and ventricular dysfunction, although complaints of palpitations are more frequent in this population. Drug treatment can help control symptoms and decrease the density and heart rate of tachycardias, but ablation is the preferred treatment, with high success rates after the first procedure, with rare complications ${ }^{4,7,8}$.

The case of an adult patient with IJRT of atypical location was reported below.

\section{CASE REPORT}

A 49-year-old man, bus driver, from Sobradinho (DF), with a history of systemic arterial hypertension (SAH) had palpitations with paroxysmal tachycardias in the last 5 years, with worsening of symptoms in the last 6 months. He reported a stronger and more symptomatic crisis while driving a bus on an important expressway in Brasilia. The electrocardiogram (ECG) performed at that time registered a narrow QRS tachycardia, a heart rate (HR) of $158 \mathrm{bpm}$, RP '> P'R and negative and well-characterized "p" waves in lower leads. (Fig. 1). The same electrocardiographic pattern was obtained on other occasions when he felt the palpitation and needed to go to the emergency room (os) to reverse the tachycardia with intravenous infusion of adenosine. The sinus rhythm ECG with HR of $68 \mathrm{bpm}$, normal AV interval and absence of ventricular pre-excitation can be observed in the Fig. 2. The transthoracic echocardiogram showed a left ventricular ejection fraction of $71 \%$, a left atrium with slightly elevated dimensions, and other walls and cavities of normal dimensions. The patient did not present significant alterations in the laboratory exams and made continuous use of indapamide, amlodipine and olmesartan to treat $\mathrm{SAH}$ and Metoprolol $25 \mathrm{mg}$, twice a day, for tachycardia controls.

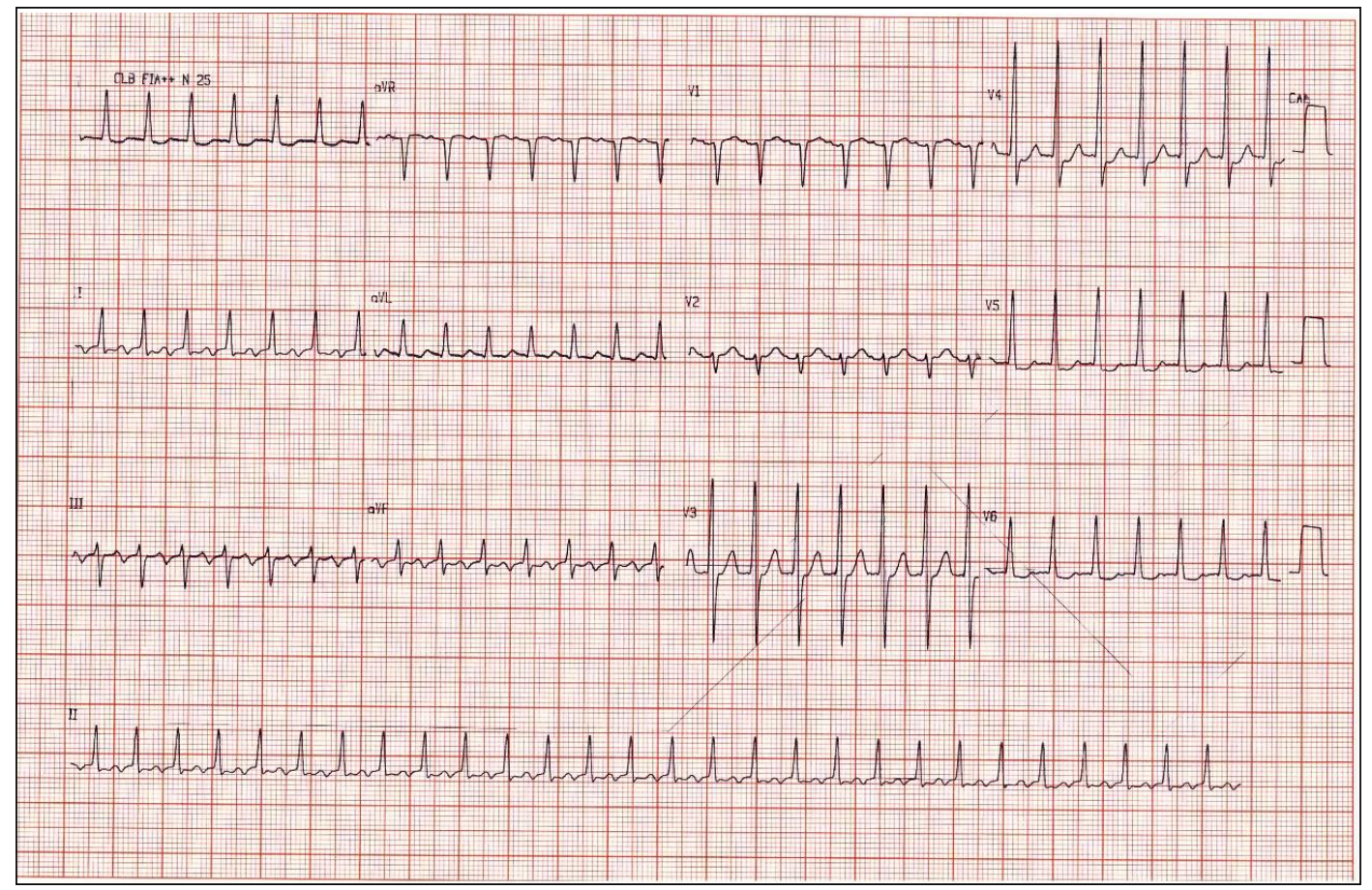

Figure 1. Tachycardia ECG 


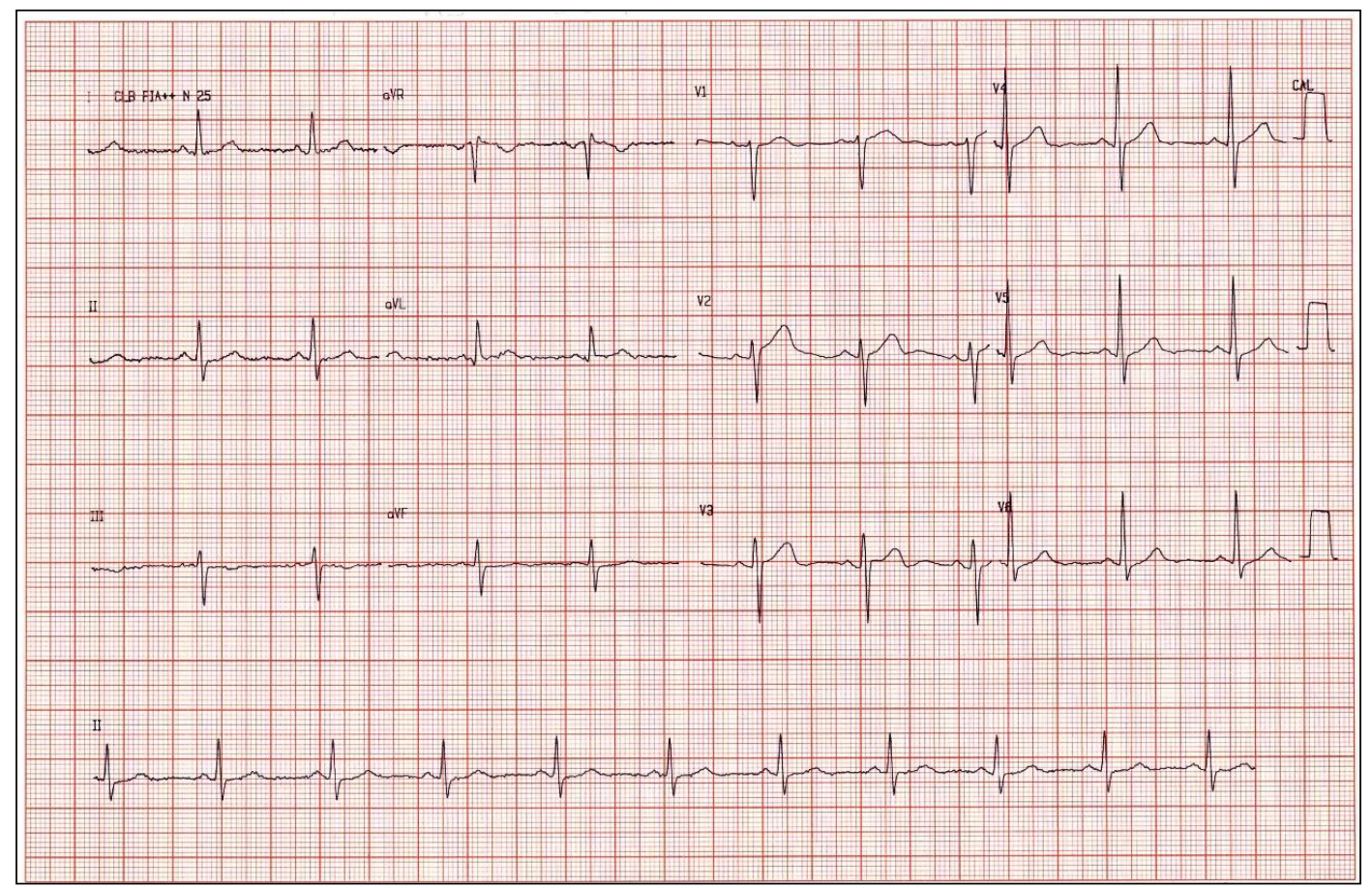

Figure 2. Baseline ECG after tachycardia reversal

After evaluation with the electrophysiologist, an electrophysiological study and ablation of tachycardia by catheter was performed in October 2019. The procedure was performed with 1 diagnostic decapolar catheter positioned in the coronary sinus after puncture of the right subclavian vein, 1 quadripolar catheter positioned at the height of the His bundle trunk and $14 \mathrm{~mm}$ dry-tip ablator catheter (Abbott ${ }^{\circledR}$ ) initially positioned in the right ventricle and later used to map potentials in a tricuspid ring to locate the accessory pathway, the last 2 catheters having been passed after puncture of the right femoral vein. The endocavitary electrical records were recorded on a polygraph (Cardian $\left.{ }^{\circledR}\right)$. During the electrophysiological study, the same tachycardia documented in previous electrocardiograms was observed, with very easy and recurrent induction. Despite the reversal after a series of ventricular stimuli, the tachycardia returned spontaneously after a few seconds, making it difficult to perform the electrophysiological study. (Fig. 3), this incessant pattern of tachycardia remained throughout the procedure. The tachycardia cycle during the examination ranged between 380 and $400 \mathrm{~ms}$ (Fig. 4). Tachycardia curtailment maneuvers were performed with stimulation at the right ventricular tip and were observed at the end of the stimulation output with a ' $\mathrm{V}-\mathrm{A}$ - V'pattern and difference between the post-pace interval (PPI) and tachycardia cycle less than $115 \mathrm{~ms}$, showing the pattern of tachycardia due to atrioventricular reentry (Fig. 5).

The accessory pathway was mapped during tachycardia, looking for the location with the smallest ventricle-atrial (AV) interval. After the location of significant precocity in the posteroseptal region of the tricuspid ring, radiofrequency ( $30 \mathrm{~W}$ and $65^{\circ} \mathrm{C}$ ) was applied for 1 minute, with interruption of tachycardia after $15 \mathrm{~s}$ of application. In sinus rhythm, programmed ventricular stimulations were performed with slow VA retrograde conduction and with decreasing characteristics with the same retrograde atrial activation sequence observed during tachycardia. During programmed atrial stimulation, tachycardia was again induced. The patient underwent a new mapping during tachycardia, performed with the aid of a long introducer to stabilize the ablation catheter, and a greater early VA was found in the right mediosseptal region, below the bundle of His (Figs. 6A e 6B). Tachycardia was stopped 3s after ablation at the site (Fig. 7). It is worth mentioning that during 1 min of application on site, there was no appearance of junctional beats or AV dissociation. Finally, new programmed atrial stimulations were performed 15 minutes after the last application, without inducing arrhythmia; there was no retrograde VA conduction at the end of the procedure.

The patient was asymptomatic in an outpatient return visit 30 days after the ablation 


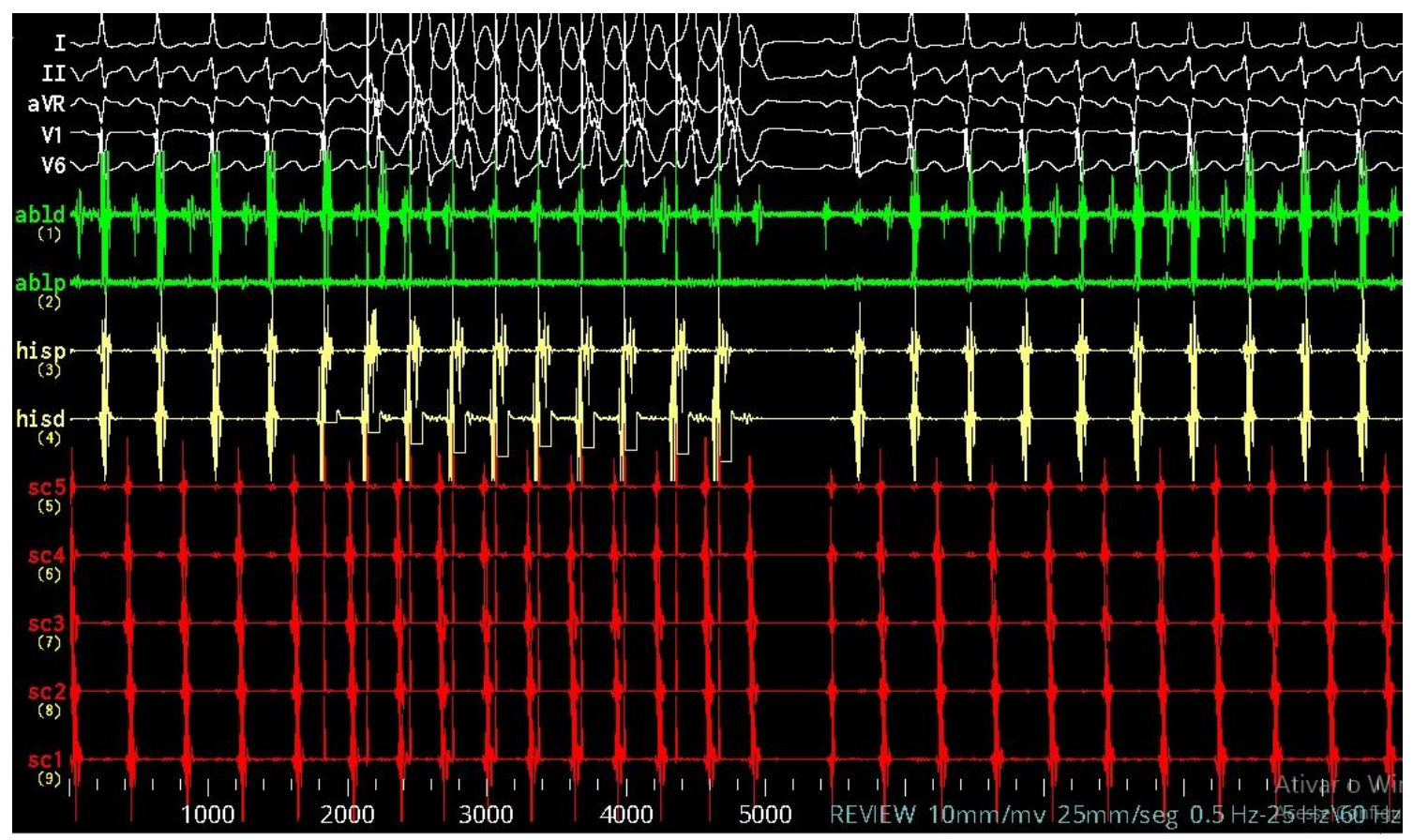

Figure 3. Tachycardia interruption after a series of ventricular pulses, followed by tachycardia reinduction after the first sinus beat. This incessant arrhythmia pattern remained throughout the electrophysiological study. SC5: proximal coronary sinus. SC1: distal coronary sinus.

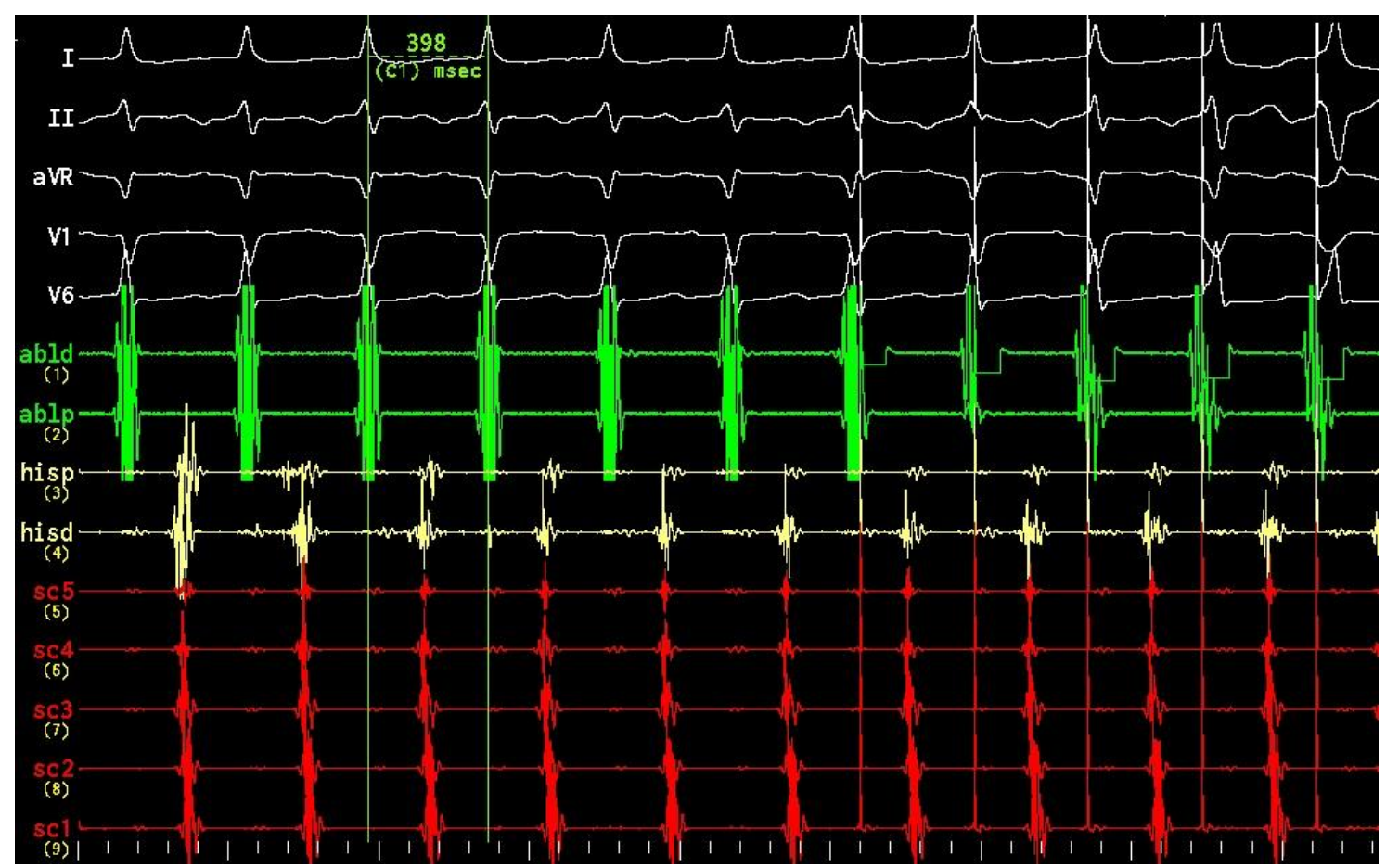

Figure 4. Tachycardia with a cycle (TCL) of $398 \mathrm{~ms}$ and start of stemming. SC5: proximal coronary sinus. SC1: distal coronary sinus 


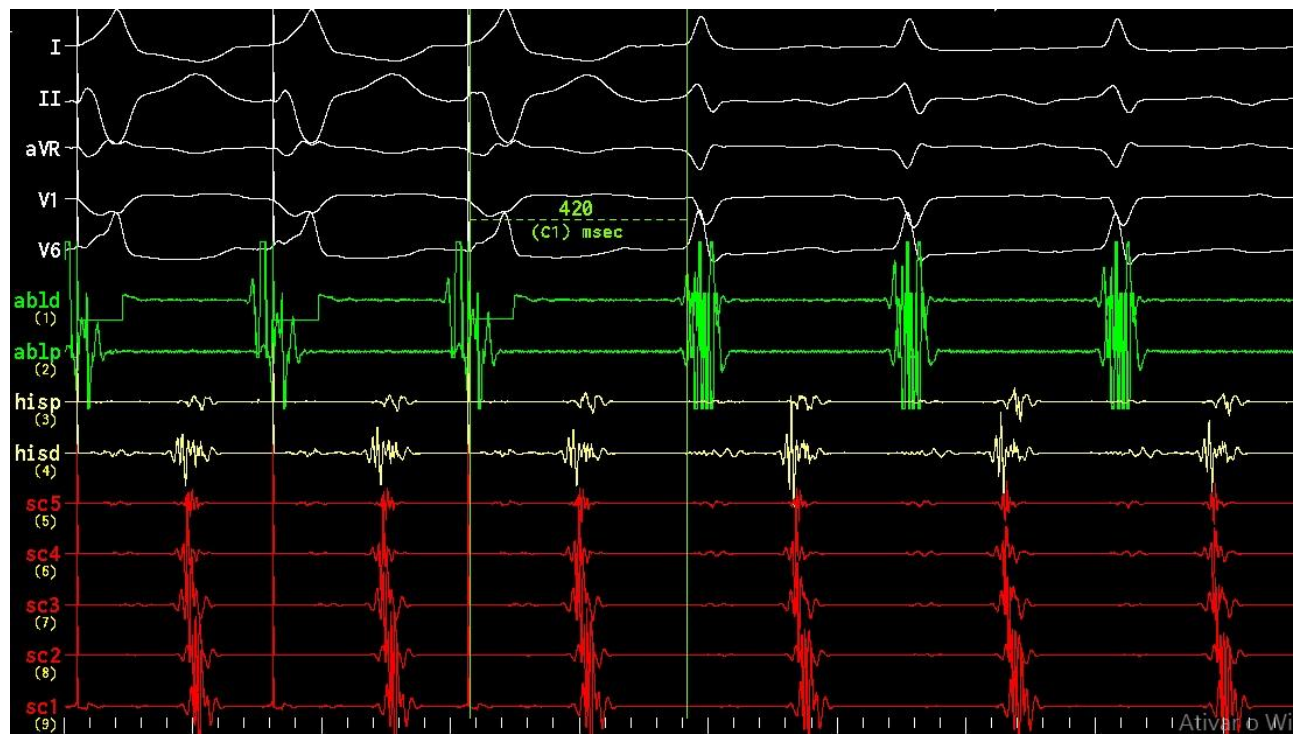

Figure 5. Tachycardia counseling with 380ms ventricular stimulation. Rails output with 420ms post-pace interval (PPI) in "V - A - V" pattern. Difference between PPI and TCL of $22 \mathrm{~ms}(420$ - 398ms). SC5: proximal coronary sinus. SC1: distal coronary sinus.

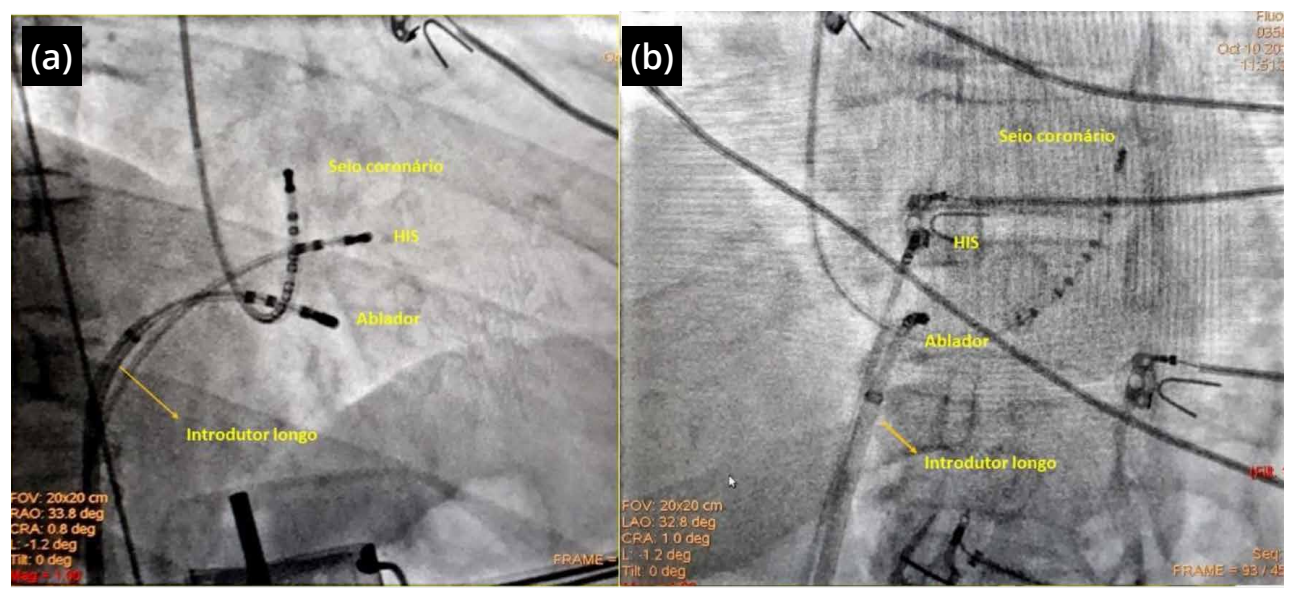

Figure 6. (a) Right anterior oblique image $30^{\circ}$ with ablating catheter in the mid-septal region D. (b) Arrangement of the catheters in the left anterior oblique $30^{\circ}$. Both images show the long introducer providing support and stabilization to the ablation catheter.

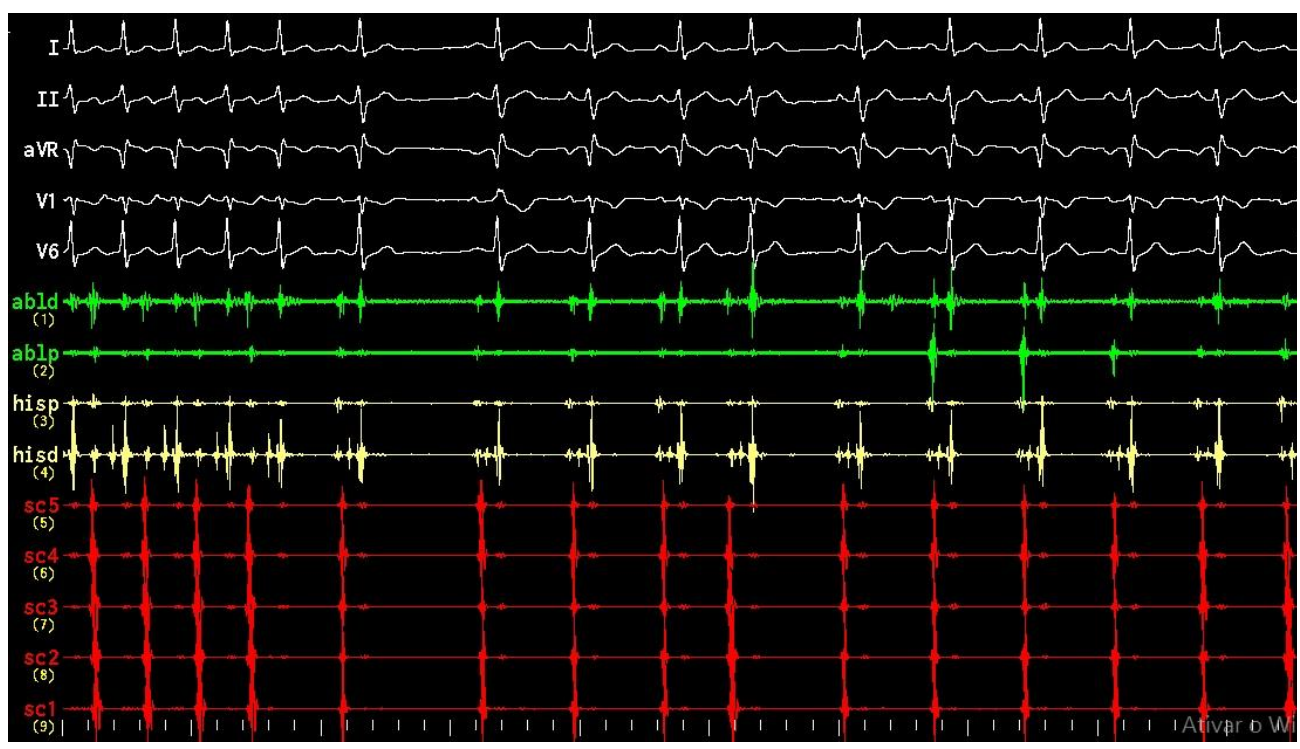

Figure 7. Tachycardia interruption during ablation. 


\section{DISCUSSION}

The treatment by ablation of IJRT has a good response and was well indicated in the case above, given its effectiveness in stopping the tachycardia episode and in the clinical improvement of the patient in the postoperative follow-up. Interestingly, the case described differs not only from the traditional epidemiology of the disease, which most commonly affects children and young people, but also in the location of the accessory pathway (usually located in the posterior or posterosseptal region of the tricuspid ring, but in this case it was located in mid-septal region).Meiltz et al. ${ }^{4}$,in a series of 49 cases of IJRT in adults, he described tachycardiomyopathy in $16 \%$ of cases. $47 \%$ of the patients presented the persistent or incessant form of tachycardia, and the rest (53\%) manifested it paroxysmally. The most common location of the accessory pathway was the right posteroseptal, however $24 \%$ of the patients evaluated had the accessory pathway in atypical locations. Of the 49 patients analyzed, only 3 had the accessory pathway in the mid-septal location, as found in the case described above ${ }^{4}$. It should also be noted that the VAs are located mainly in the tricuspid ring, stabilizing the ablation catheter to perform the procedure can be challenging, especially in an area close to the trunk of the bundle of His, such as the mid septal region. In this report, the use of the long introducer to stabilize the ablation catheter was decisive for the success of VA ablation.

\section{CONCLUSION}

This report demonstrated the importance not only of including IJRT among the differential diagnoses of supraventricular tachycardia even in adults (especially those with a long RP 'interval on the tachycardia ECG), but also of the need for an adequate mapping in different locations, not only from the right posterosseptal region, for the correct location of the AV in these cases. It should be added that the use of the long sheath helped both in the mapping and in the stabilization of the ablation catheter during the application of the radiofrequency.

Ablation is the treatment of choice at IJRT and, despite the location of the AV in this case, the procedure was successful. Therefore, treatment by ablation should be indicated in these situations.

\section{REFERENCES}

1. Coumel P, Cabrol C, Fabiato A, et al. Tachycardie permanente par rythme réciproque. Preuves du diagnostic par stimulation auriculaire et ventriculaire. Arch Mal Coeur. 1967;60:1830-64.

2. Coumel P. Junctional reciprocating tachycardias. The permanent and paroxysmal forms of A-V nodal reciprocating tachycardias. $J$ Electrocardiol. 1975;8(1):79-90. https://doi.org/10.1016/s0022-0736(75)80043-4

3. Critelli G, Gallagher J], Thiene G, Rossi L. The permanent form of junctional reciprocating tachycardia. In: Benditt DG, Benson DW, eds. Cardiac Preexcitation Syndromes. Springer, Boston: Martinus Nijhoff; 1986. pp 233-53. https://doi.org/10.1007/978-1-4682-7526-5_11

4. Meiltz A, Weber R, Halimi F, Defaye P, Boveda S, Tavernier R, et al. Permanent form of junctional reciprocating tachycardia in adults: peculiar features and results of radiofrequency catheter ablation. Europace. 2006;8(1):21-8. https://doi.org/10.1093/europace/euj007

5. Critelli G. Recognizing and managing permanent junctional reciprocating tachycardia in the catheter ablation era. J Cardiovasc Electrophysiol. 1997;8(2):226-36. https://doi.org/10.1111/j.1540-8167.1997.tb00784.x

6. Ticho BS, Saul JP, Hulse JE, De W, Lulu J, Walsh EP. Variable location of accessory pathways associated with the permanent form of junctional reciprocating tachycardia and confirmation with radiofrequency ablation. Am J Cardiol. 1992;70(20):1559-64. https://doi. org/10.1016/0002-9149(92)90457-a

7. Dorostkar PC, Silka MJ, Morady F, Dick M. Clinical course of persistent junctional reciprocating tachycardia. J Am Coll Cardiol. 1999;33(2):366-75. https://doi.org/10.1016/s0735-1097(98)00590-7

8. Packer DL, Bardy GH, Worley SJ, Smith MS, Cobb FR, Coleman RE, et al. Tachycardia-induced cardiomyopathy: a reversible form of left ventricular dysfunction. Am J Cardiol. 1986;57(8):563-70. https://doi.org/10.1016/0002-9149(86)90836-2 